\title{
Papel de los nuevos anticoagulantes orales en el tratamiento de la enfermedad coronaria
}

\section{The role of the new oral anticoagulants in the treatment of coronary disease}

\author{
Hernando del Portillo ${ }^{\mathrm{a}, \mathrm{b}}$, Juan J. Badimón ${ }^{\mathrm{b}, \mathrm{c}}$, Jaime R. Cabrales ${ }^{\mathrm{a}, \mathrm{b}}$ y Darío Echeverri ${ }^{\mathrm{a}, \mathrm{b}, *}$ \\ a Servicio de Hemodinamia e Intervencionismo Cardiovascular, Fundación Cardiolnfantil - Instituto de Cardiología, Bogotá, \\ Colombia \\ ${ }^{\mathrm{b}}$ Facultad de Medicina Universidad del Rosario, Bogotá, Colombia \\ c Atherothrombosis Research Unit, The Zena and Michael A. Wiener Cardiovascular Institute, Icahn School of Medicine at Mount \\ Sinai, New York, United States
}

Recibido el 30 de septiembre de 2016; aceptado el 6 de octubre de 2016

Disponible en Internet el 17 de noviembre de 2016

Los nuevos anticoagulantes orales llegaron para quedarse en la prevención de ataques cerebrovasculares isquémicos en pacientes con fibrilación auricular no valvular. Varios estudios clínicos han establecido su eficacia y seguridad ${ }^{1-3}$. Sin embargo, el papel que pueden tener en otras patologías, como la enfermedad coronaria, no está bien estudiado y todavía existen varias e importantes preguntas sin responder. Una de estas es su papel en la prevención secundaria de la enfermedad coronaria, dado que a pesar de un tratamiento médico óptimo y la doble terapia antiplaquetaria, el riesgo de reinfarto ha disminuido tan solo un 30\%, fenómeno posiblemente explicado por otros factores relacionados, como el metabolismo lipídico, el estado inflamatorio y el estado protrombótico en el que el factor $x$ activado (Xa), tiene un rol fundamental al generar la conversión de protrombina inactiva a trombina, la cual es el agonista más potente para la agregación plaquetaria ${ }^{4,5}$. El segundo interrogante está relacionado con los pacientes que tienen fibrilación auricular no valvular y enfermedad coronaria que requieren implante de un stent, en quienes los nuevos

\footnotetext{
* Autor para correspondencia.

Correo electrónico: decheverri@cardioinfantil.org (D. Echeverri).
}

anticoagulantes orales podrían llegar a ser una alternativa en combinación con antiagregantes plaquetarios.

Actualmente, en Colombia se cuenta con dos tipos de nuevos anticoagulantes orales, los inhibidores directos del factor $x$ activado (rivaroxabán y apixabán) y los inhibidores directos de la trombina (dabigatrán), que pueden ser una nueva herramienta terapéutica para responder a estos interrogantes. Los inhibidores del factor Xa suprimen la síntesis de trombina de una manera indirecta al inhibir este factor, mientras que los antitrombínicos directos inhiben la actividad de la trombina.

\section{Primer escenario: prevención secundaria en enfermedad coronaria}

La trombosis coronaria depende de la actividad plaquetaria y su integración con las vías de la coagulación, donde los factores dependientes de vitamina K (II,VII, IX y x) ejercen un papel muy importante ${ }^{6}$, razón por la cual hace algunos años los antagonistas de vitamina K (AVK) fueron estudiados para la prevención secundaria de eventos cardiovasculares, reduciendo de manera significativa los episodios de ataques cerebrovasculares e infarto del miocardio. Los enormes beneficios de esta clase de agentes tenían una desventaja, el aumento significativo del sangrado ${ }^{7}$, probablemente 
asociado a la dificultad de obtener y mantener niveles de INR en rangos terapéuticos, debido a las múltiples interacciones con otros medicamentos e incluso con la dieta y el inicio lento de acción. Todos esos condicionantes son parte de las razones por las que actualmente los AVK no se consideran medicamentos de primera elección en la prevención y el tratamiento de eventos cardiovasculares.

Estudios recientes han evaluado las posibilidades terapéuticas de los nuevos anticoagulantes orales para la prevención secundaria en pacientes con enfermedad coronaria. Los más destacados son el APPRAISE-2 con apixabán ${ }^{8}$ y el ATLAS-ACS 2 TIMI 51 con rivaroxabán ${ }^{9}$, que tuvieron como objetivo primario disminuir la muerte cardiovascular, el infarto del miocardio y el ataque cerebrovascular en pacientes con enfermedad coronaria conocida. Infortunadamente, los resultados fueron poco alentadores. El APPRAISE-2, fue terminado de manera prematura debido a exceso de sangrado. El ATLAS-ACS 2 TIMI 51 fue positivo, pero los resultados son difíciles de explicar con base en el punto de vista farmacológico, ya que las dosis bajas de rivaroxabán de $2,5 \mathrm{mg}$ cada 12 horas disminuyeron la mortalidad cardiovascular pero no el infarto de miocardio (siendo esta la principal causa de muerte cardiovascular), mientras que dosis de $5 \mathrm{mg}$ cada 12 horas reducían las tasas de infarto de miocardio pero no la mortalidad cardiovascular. Además, ambas dosis demostraron aumento en el sangrado frente al grupo control de referencia.

Pero la idea que consideramos importante resaltar y nos llama la atención es en el ámbito de la prevención secundaria, ya que el beneficio estuvo conducido principalmente por los resultados luego del primer año de tratamiento, momento en el cual la mayoría de los pacientes descontinúan la terapia antiplaquetaria dual, por lo que nos preguntamos ¿Será el rivaroxabán el próximo medicamento de elección para la prevención secundaria en la enfermedad coronaria? Para tratar de resolver esta pregunta se encuentra en curso el estudio fase ॥ GEMINI-ACS-10 ${ }^{10}$, el cual usará dosis de $2,5 \mathrm{mg}$ cada $12 \mathrm{~h}$ de rivaroxabán en reemplazo del ácido acetil salicílico combinado con clopidogrel y ticagrelor buscando una doble vía de acción, y cuyo objetivo principal será evaluar la seguridad en términos de sangrado medido por la escala TIMI.

\section{Segundo escenario: triple terapia con nuevos anticoagulantes orales}

Con los cambios en la pirámide poblacional que conllevan aumento en la edad de los pacientes, y el incremento en la incidencia de fibrilación auricular y claro beneficio de la anticoagulación con los nuevos anticoagulantes orales en pacientes con fibrilación auricular, cada vez es más frecuente el escenario clínico en el cual los pacientes requieren antiagregación dual y anticoagulación. Acerca de la terapia triple en pacientes con fibrilación auricular y enfermedad coronaria más intervención coronaria percutánea (ICP), en el momento se encuentran en curso varios estudios con diferentes tipos y dosis de nuevos anticoagulantes orales, como lo son el RE-DUAL PCl con dabigatrán, el PIONEERAF con rivaroxabán y el AUGUSTUS con apixabán ${ }^{11}$, los cuales evaluarán la combinación de estos nuevos anticoagulantes orales con los diferentes inhibidores del receptor
$P_{2} Y_{12}$. Estos estudios son bastante lógicos si se analizan tres aspectos: 1) el mayor beneficio de los nuevos anticoagulantes orales frente a los AVK es el menor riesgo de sangrado; 2) algunos nuevos anticoagulantes orales ya han sido estudiados para la prevención secundaria; y 3) los nuevos anticoagulantes orales ya cuentan con antídotos, algunos en fase III de investigación.

\section{Nuevos y posibles escenarios de los nuevos anticoagulantes orales en la enfermedad coronaria}

En casos de insuficiencia cardiaca de origen isquémico y fracción de eyección reducida, se encuentra en curso el estudio fase III COMMANDER-HF ${ }^{12}$, que se basa en la teoría de la trombina como elemento crítico para empeorar la falla cardiaca, de ahí que el uso del rivaroxabán con dosis de $2,5 \mathrm{mg}$ cada 12 horas más terapia antiplaquetaria dual o sencilla, evaluará el desenlace compuesto de mortalidad por cualquier causa, infarto de miocardio y ataque cerebrovascular. En este mismo contexto, pero con la presencia de trombos ventriculares izquierdos, ya se han reportado casos de uso de los nuevos anticoagulantes orales en los que se ha observado resolución completa del trombo durante el seguimiento ecocardiográfico ${ }^{13}$.

Por último, en la enfermedad coronaria ectásica se ha comprobado la presencia de flujo coronario lento, lesión endotelial y estado pro-inflamatorio que genera un ambiente de hipercoagulabilidad ${ }^{14}$; cumpliendo entonces con las características aun aceptadas de la tríada de Virchow, estos pacientes tendrán una predisposición alta a la formación de trombos y recurrencia de eventos isquémicos miocárdicos. En esta enfermedad coronaria ectásica con flujos lentos y trombosis coronaria, se han utilizado diferentes estrategias de tratamiento como la trombectomía manual más anticoagulación, trombolíticos intracoronarios, ácido acetil salicílico más warfarina, inhibidores de la glicoproteína $\mathrm{II}_{\mathrm{b}} / \mathrm{II}_{\mathrm{a}}$ y stents liberadores de medicamento, mostrando resultados controversiales ${ }^{15}$. Por lo anterior, la dosis de rivaroxabán de $2,5 \mathrm{mg}$ cada 12 horas podría ser una opción farmacológica interesante y viable para este grupo de pacientes, dado que aún no se conoce un tratamiento específico para el manejo de esta patología. El uso de los nuevos anticoagulantes orales en este contexto, podría ser un campo para explorar en la investigación clínica.

\section{Bibliografía}

1. Patel MR, Mahaffey KW, Garg J, Pan G, Singer DE, Hacke W, et al. Rivaroxaban versus warfarin in nonvalvular atrial fibrillation. N Engl J Med. 2011;365:883-91.

2. Granger CB, Alexander JH, McMurray JJV, Lopes RD, Hylek EM, Hanna M, et al. Apixaban versus warfarin in patients with atrial fibrillation. N Engl J Med. 2011;365:981-92.

3. Connolly SJ, Ezekowitz MD, Yusuf S, Eikelboom J, Oldgren J, Parekh A, et al. Dabigatran versus warfarin in patients with atrial fibrillation. N Engl J Med. 2009;361:1139-51.

4. Vilahur G, Badimon JJ, Bugiardini R, Badimon L. Perspectives: The burden of cardiovascular risk factors and coronary heart disease in Europe and worldwide. Eur Heart J. 2014;16 suppl A:A7. 
5. Santos-Gallego CG, Badimon L, Badimón JJ. Perspectives: Direct and specific inhibition of factor $\mathrm{Xa}$ : an emerging therapeutic strategy for atherothrombotic disease. Eur Heart J. 2014;16 suppl A:A56.

6. De Caterina R, Husted S, Wallentin L, Andreotti F, Arnesen $\mathrm{H}$, Bachmann $\mathrm{F}$, et al. Oral anticoagulants in coronary heart disease (Section IV). Position paper of the ESC Working Group on Thrombosis-Task Force on Anticoagulants in Heart Disease. Thromb Haemost. 2016;115:685-711.

7. Hurlen M, Abdelnoor M, Smith P, Erikssen J, Arnesen H. Warfarin, aspirin, or both after myocardial infarction. N Engl J Med. 2002;347:969-74.

8. Alexander JH, Lopes RD, James S, Kilaru R, He Y, Mohan P, et al. Apixaban with antiplatelet therapy after acute coronary syndrome. N Engl J Med. 2011;365:699-708.

9. Mega JL, Braunwald E, Wiviott SD, Bassand J-P, Bhatt DL, Bode $C$, et al. Rivaroxaban in patients with a recent acute coronary syndrome. N Engl J Med. 2012;366:9-19.

10. Povsic TJ, Roe MT, Ohman EM, Steg PG, James S, Plotnikov A, et al. A randomized trial to compare the safety of rivaroxaban vs aspirin in addition to either clopidogrel or ticagrelor in acute coronary syndrome: The design of the GEMINI-ACS-1 phase II study. Am Heart J. 2016;174:120-8.

11. Heidbuchel H, Verhamme P, Alings M, Antz M, Diener H-C, Hacke W, et al. Updated European Heart Rhythm Association Practical
Guide on the use of non-vitamin $K$ antagonist anticoagulants in patients with non-valvular atrial fibrillation. Europace. 2015;17:1467-507.

12. Zannad F, Greenberg B, Cleland JGF, Gheorghiade M, van Veldhuisen DJ, Mehra MR, et al. Rationale and design of a randomized, double-blind, event-driven, multicentre study comparing the efficacy and safety of oral rivaroxaban with placebo for reducing the risk of death, myocardial infarction or stroke in subjects with heart failure and significant coronary artery disease following an exacerbation of heart failure: the COMMANDER HF trial. Eur J Heart Fail. 2015;17: 735-42.

13. Mano $Y$, Koide K, Sukegawa H, Kodaira M, Ohki T. Successful resolution of a left ventricular thrombus with apixaban treatment following acute myocardial infarction. Heart Vessels. 2016;31:118-23.

14. Brunetti ND, Salvemini G, Cuculo A, Ruggiero A, De Gennaro L, Gaglione A, et al. Coronary artery ectasia is related to coronary slow flow and inflammatory activation. Atherosclerosis. 2014;233:636-40.

15. Eitan A, Roguin A. Coronary artery ectasia: new insights into pathophysiology, diagnosis, and treatment. Coron Artery Dis. 2016;27:420-8. 